\title{
Facility Management - Erster Hauptstudiengang an der Technischen Fachhochschule Wildau erfolgreich gestartet
}

\author{
Dietrich Reiblich
}

\section{Einleitung}

Elf Studenten des Matrikels W2/97 begannen im Fruihjahr 1999 hochmotiviert den ersten Hauptstudiengang Facility Management am Fachbereich Ingenieurwesen/Wirtschaftsingenieurwesen. Die Fachhochschule Wildau gehört damit zu den wenigen Hochschulen in Deutschland, die einen solchen Studiengang bereits anbieten. Die Nachfrage nach gut ausgebildeten Facility Managern ist groß und wird mit Sicherheit in den nächsten Jahren noch steigen. Ein gutes Gefuihl für die Studierenden, wenn man weiß, daß die Unternehmen im Dienstleistungsmarkt verzweifelt nach jungen Führungskräften für diese Disziplin suchen.

Das Studium an der TFH Wildau orientiert sich konsequent an der vom Deutschen Verband für Facility Management e.V. erarbeiteten Richtlinie Nr. 610 „FM-Studiengänge“ [1] mit der ein wichtiger Beitrag zur Vereinheitlichung der Ausbildung des Facility Managers im deutschsprachigem Raum geleistet wurde. Damit können Auszubildende und nachfragende Unternehmen sicher sein, daß den Ausbildungsanforderungen an die integrierende Fachdisziplin Facility Management durch die Hochschule voll entsprochen wird.

\section{Facility Management (FM) - eine Managementdisziplin}

Die seit Jahren spürbare Inflation der Managementbegriffe hat eine Deflation des Anspruchs zur Folge, d. h. es wird immer weniger reflektiert was es heißt zu managen, denn die Aktion bestimmt zunehmend das Sein und leider auch das Bewußtsein. Auch der Begriff „Facility Management“ läuft Gefahr, vom (sinnbildlich gesprochen) Degradieren des Managements erfaßt zu werden. So ist für die einen FM nur ,eine dieser neumodischen Bezeichnungen für das was wir schon lange tun“ z.B. Hausmeister, Instandhalter, Gebäudereiniger usw. für andere vordergründig ein Reputationsbegriff. Aus dieser Fehleinschätzung resultiert letztlich auch die besonders in Deutschland verbreitete Identifizierung des Facility Management mit Dienstleistungen rund um das Gebäude schlechthin.

Tatsächlich ist FM eine unternehmerische Aufgabe im gesamten Lebenszyklus ${ }^{1}$ der Facilities ${ }^{2}$, die höchste Ansprüche an die Managementqualitäten der Führungskräfte stellt. Facility Management verlangt ganzheitliches Denken und eine an den Zielen der FM-Organisation orientierte Managementphilosophie.

„Ganzheitlichkeit ist ohne Sinnbezug undenkbar, denn das Wesen von Zusammenhängen im Wechselspiel von Teilen und Ganzem erschließt sich erst über die Konstruktion eines Sinnes.“ [2]
Der Sinnbezug im FM wird geprägt durch bewußtes „Dienen“- hier als eine Leistung mit der das wirtschaftliche Ergebnis aus der Nutzung der Facilities verbessert und gleichzeitig das Business der Nutzer von Facilities unterstiitzt wird.

Der Facility Manager versteht sich damit als Dienstleister und /oder Consulter für Eigentümer und Nutzer von Facilities. Das Management des Dienstleistungs- oder Consultingprozesses hat wie bei anderen Führungsprozessen auch jeweils eine strategische, normative und operative Dimension (Bild 1), deren Gewichtung in Abhängigkeit der Lebenszyklusphase der Facilities zu sehen ist, in der die FM-Organisation tätig wird.

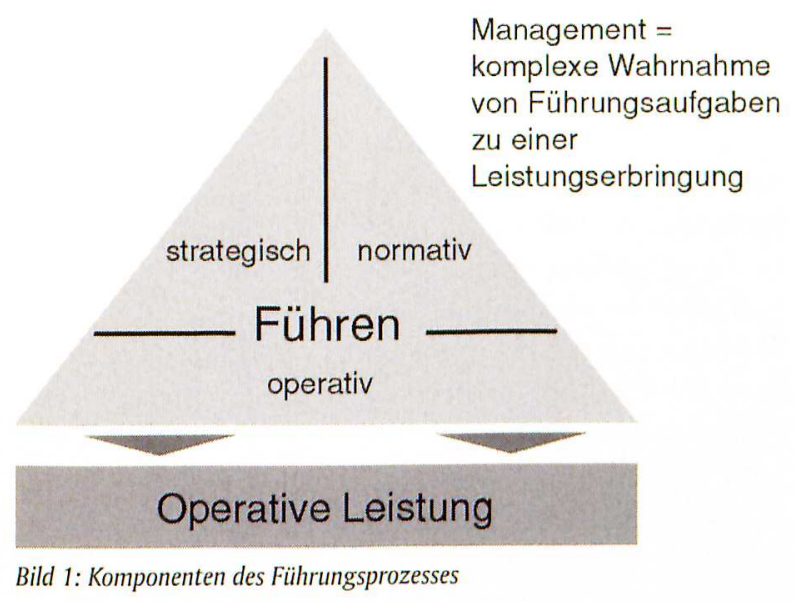

Typisch fuir den Managementkreis im FM (Bild 2) ist, daß dieser auf der integrierten Datenbasis der Facilities und einer an den objektkonkreten Bedürfnissen orientierten Aufbau- und Ablauforganisation aufsetzt.

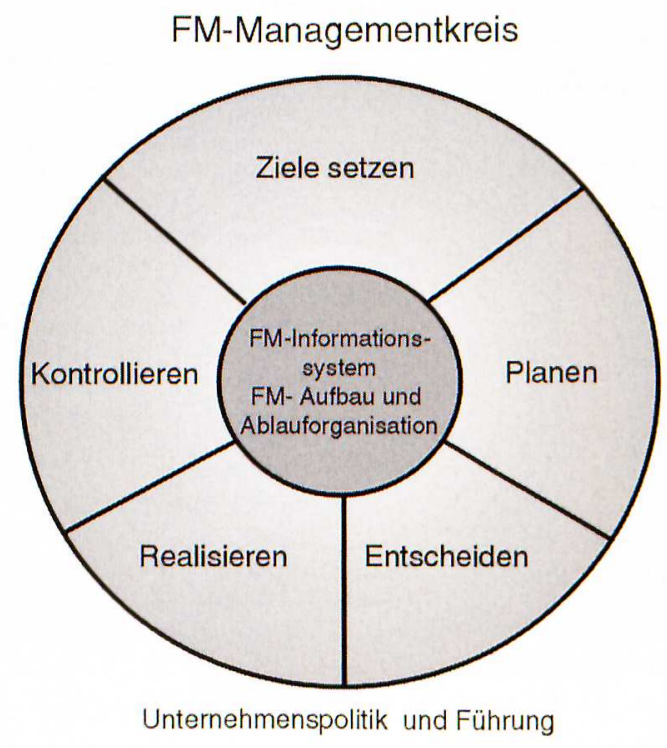

Bild 2: Managementkreis in FM 
Diese FM-Organisation wiederum wird bestimmt durch eine Vielzahl von Einflußfaktoren auf den Facility Managementprozeß, klassifizierbar in gesellschaftliche, makrostrukturelle und mikrostrukturelle Einflüsse. [3] Der Facility Managementprozeß ist von außerordentlicher Dynamik gekennzeichnet, bei dem es darauf ankommt, daß die FM-Organisation, aufbauend auf ihrer jeweiligen Kernkompetenz, sich an den objektkonkreten Bedingungen orientiert und den sich verändernden Leistungsschwerpunkten und Bedürfnissen schnell anpassen kann.

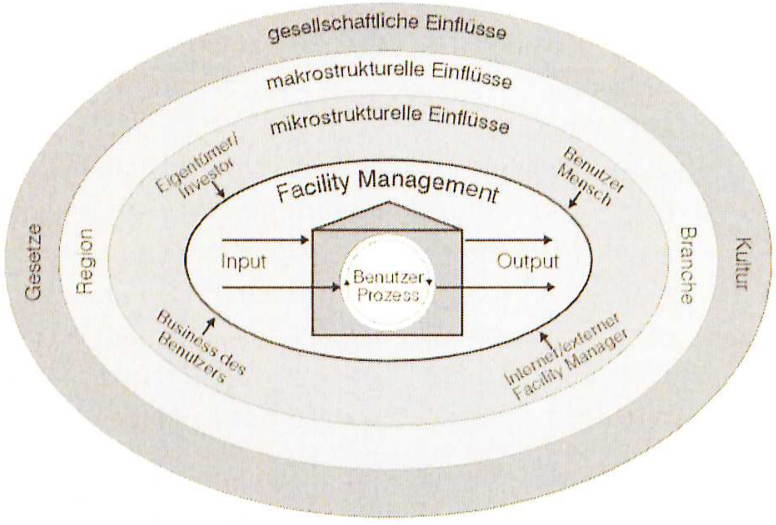

Bild 3: FM im Spannungsfeld von Major Players und Kerngeschäft

Aus seiner Stellung gegenüber den Major Players ${ }^{3}$ der Anlagenwirtschaft (Bild 3) wird auch das komplexe Anforderungsbild an den Facility Manager erahnbar. Der Facility Manager benötigt zur Führung der integrierten FM-Prozesse ein breites Spektrum an Kenntnissen in deren Mittelpunkt eine ausgeprägte soziale und Führungskompetenz steht. Diese Fähigkeiten müssen auf einem soliden Wissen in den Bereichen der Anlagenplanung und des Anlagenbetriebes sowie der Prozeßorganisation aufbauen. Zur Durchfuihrung dieser Aufgaben und zur Erfïllung der Anforderungen ist das Beherr-

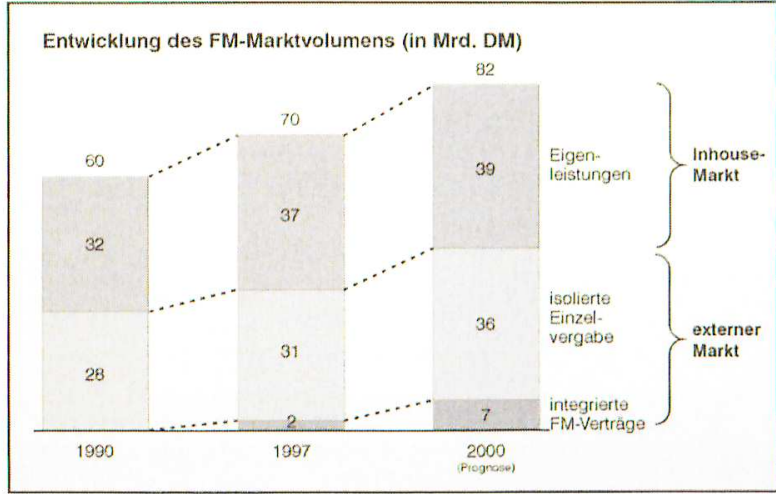

Bild 4: Facility Management ein TOP-Wachstums-Markt

schen moderner Managementwerkzeuge sowie der sichere Umgang mit dem Recht, insbesondere bei der Gestaltung der Liefer- und Leistungsbeziehungen zwischen den Akteuren der Anlagenwirtschaft erforderlich. Diese Fähigkeiten zu entwickeln ist eine der Kernaufgaben in der Ausbildung an der TFH Wildau, wohl wissend, daß hier nur die Grundlagen vermittelt werden können, auf die der Absolvent als Facility Manager beim objektkonkreten Einsatz aufbauen kann.

\section{Tendenzen der FM-Marktentwicklung}

Wenige Märkte wachsen so kontinuierlich wie nun seit Jahren schon das Potential des FM-Nachfragemarktes (Bild 4) und alle Prognosen wurden bislang übertroffen. Auch wenn es sich dabei zum erheblichen Teil um eine Umschichtung des vorhandenen Marktvolumens handelt, so sind die Chancen für FM-Consulter und FMDienstleister in dieser dynamischen Marktentwicklung außerordentlich hoch. Diese lassen sich allerdings nur dann nutzen, wenn es dem FM-Leistungsanbieter überzeugend d.h. meßbar gelingt, mit seiner Tätigkeit Nut-

\section{Gebäudeart}

Schulen und Kindergärten

Krankenhäuser

Hallenbäder

Sporthallen

Freibäder

Verkehrsanlagen

Produktionsgebäude

Büro und Verwaltungsgebäude

\section{Baufolgekosten in Prozent der Baukosten p.a.}

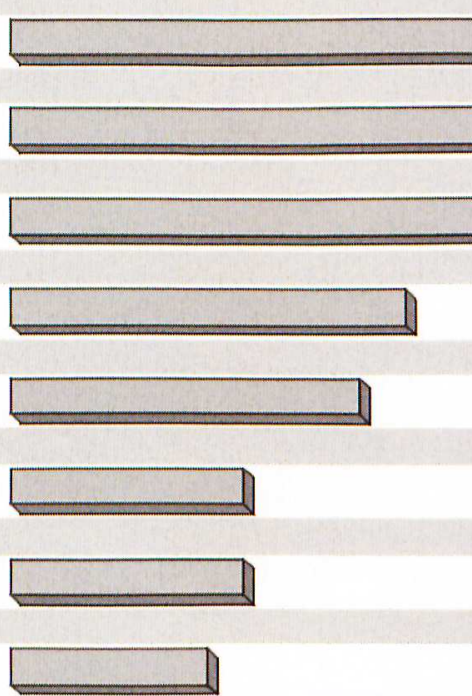

Überschreitung der Erstellungskostenschwelle nach .

$\begin{array}{lll}31 \% & \rightarrow & 3-4 \text { Jahren } \\ 26 \% & \rightarrow & 4 \text { Jahren } \\ 21 \% & -> & 4-5 \text { Jahren } \\ 17 \% & \rightarrow & 5-6 \text { Jahren } \\ 15 \% & -> & 6-7 \text { Jahren } \\ 10 \% & \rightarrow & 10 \text { Jahren } \\ 10 \% & \rightarrow & 10 \text { Jahren } \\ 8,5 \% & \rightarrow & 11-12 \text { Jahren }\end{array}$


zen beim Kunden zu erzeugen; die Ansatzpunkte dazu sind außerordentlich vielfältig.

Allein wenn man die jährlichen Nutzungskosten für verschiedene Gebäudearten betrachtet (Bild 5), wird deutlich was es bedeutet, wenn nur 1\% dieser Kosten (bei einem durchschnittlichen Anteil der Liegenschaften und Gebäude am Anlagenbestand der deutschen Industrie von ca. 30\%) durch FM eingespart werden können. Die Erschließung von Einsparpotentialen im Lebenszyklus der Facilities ist es, was den Markt vorantreibt und die Anbietergruppen (Bild 6) mobilisiert.

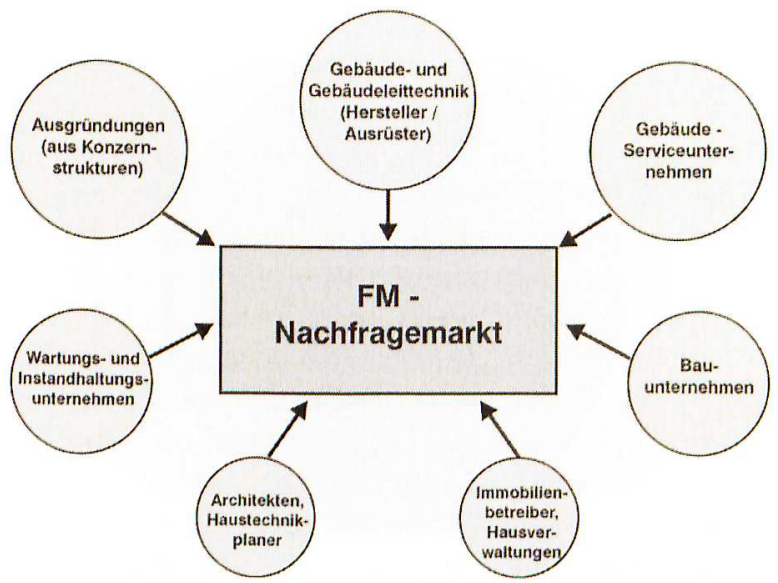

Bild 6: Anbietergruppen für den FM-Nachfiagemarkt

Für einige von ihnen entwickelt sich FM zu einem neuen Kerngeschäft (z.B. Architekten, Haustechnikplaner,...) für andere bedeutet FM vorwiegend Qualifikations-, Struktur- und Leistungsanpassung bzw. -neuorientierung (z.B. Hausverwaltungen, Gebäude- Serviceunternehmen,...).

Aus den nachfolgend thesenhaft beschriebenen Tendenzen der Entwicklung des FM kann das Profil für Absolventen des Facility Management-Studiums abgeleitet werden, das auch durch die konkreten Anforderungen der FM-Unternehmen mitbestimmt wird.
1. Die rasante Entwicklung der IT-Technologien befördert die kostengiinstige Erarbeitung der Grundlagen für den FM-Prozeß, die in erster Linie in der kompromißlosen Datenintegration der Facility-Objektbeschreibung (Bild 7) und dem Aufbau von FM-Informationssystemen (in der Regel als Kombination von Datenbank und CAD-Grafik) (Bild 8) bestehen.

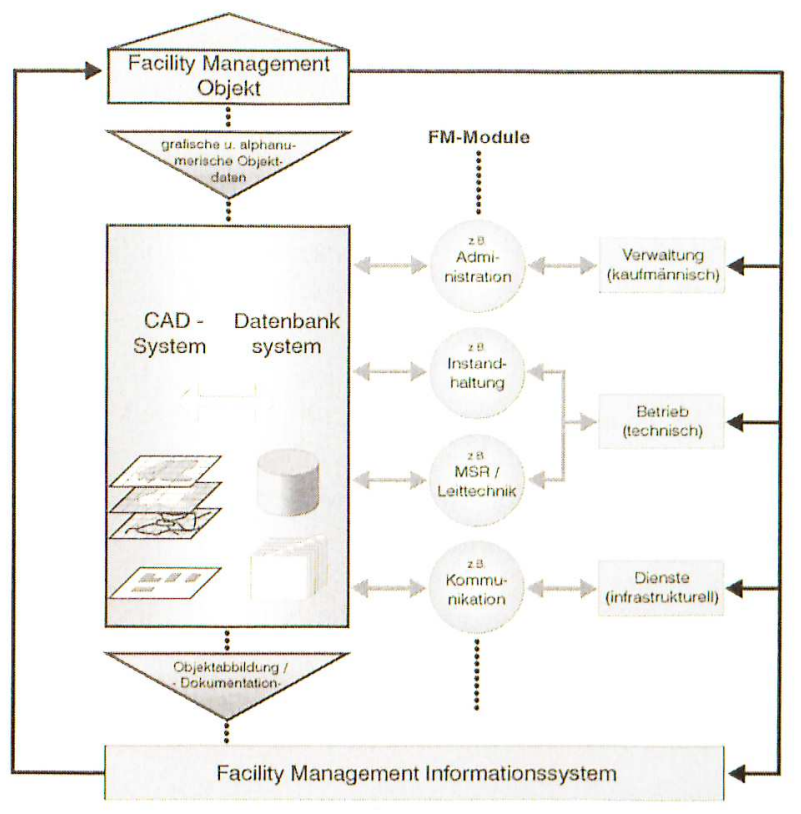

Bild 8: Abbildung der Facilities im FM-Informationssystem

Erst mit Vorliegen der Echtzeitinformationen d.h. der „Dokumentation der Wahrheit" lassen sich wesentliche Einsparpotentiale der Facilities erschließen. Gleichzeitig wächst die Tendenz zum Fernmanagement bei Organisationen mit geografisch verteilten Liegenschaften und Objekten. Datenbestände der Facilities großer Organisationen werden zentral verwaltet, in der Zentrale die aktuellen Zustände der Facilities überwacht und Handlungsstrategien durch die FM-Führungsorganisation entwickelt.

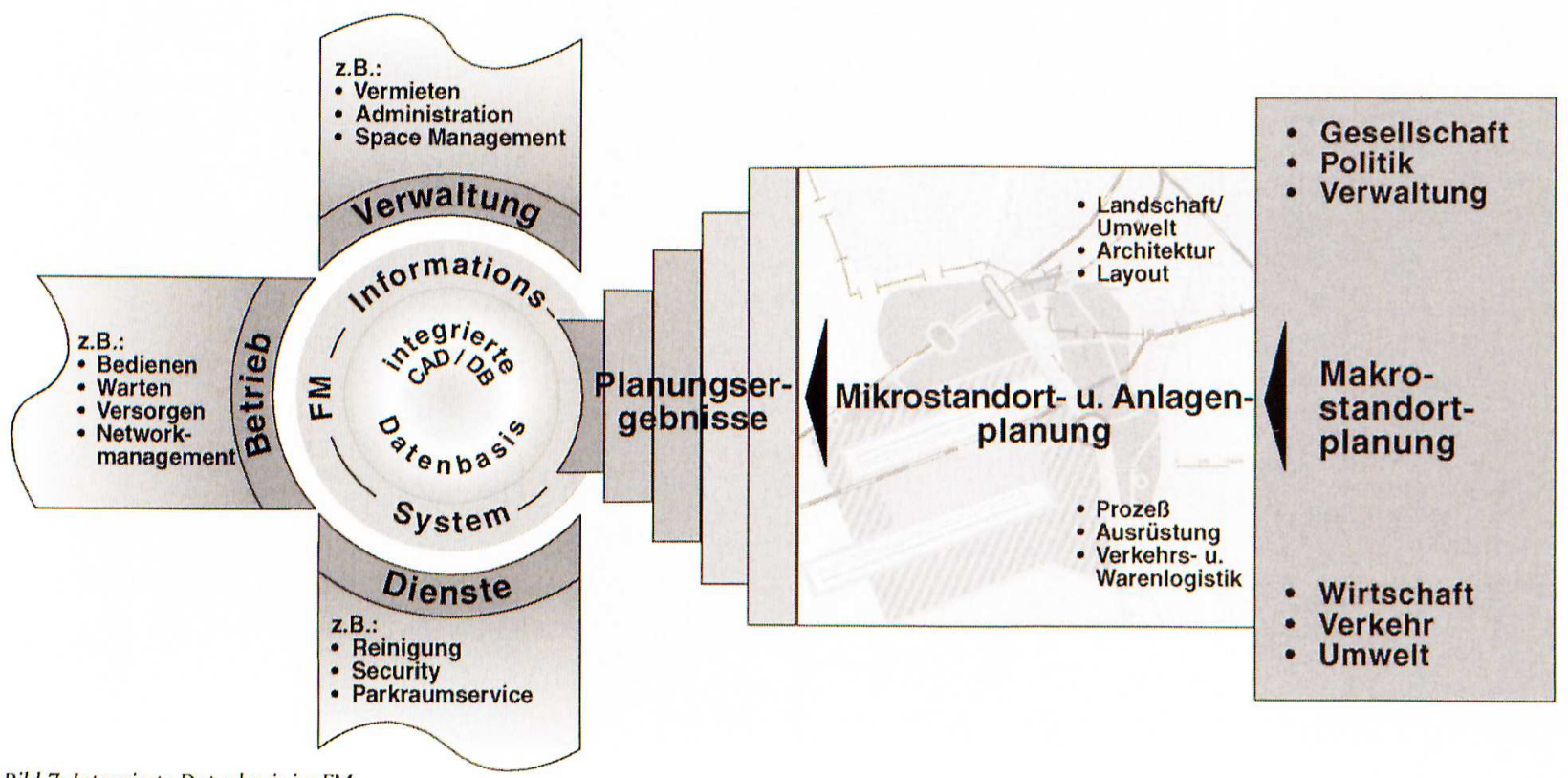


2. Es wächst die Erkenntnis, daß z.B. bei Neubauplanung, Umplanung und Rekonstruktionen dann ein hoher FM-Nutzen generiert werden kann, wenn der Facility Manager von Anfang an in den Planungs- und Realisierungsprozeß einbezogen wurde (Bild 9). Damit werden sich letztlich mit der Hinwendung zum FM neue Berufsbilder bei Architekten, Projektmanagern, beratenden Ingenieuren u.a. ergeben. Die den Planungsprozeß begleitenden Facility Manager werden mit solchen Leistungen wie nutzungsorientierte Wirtschaftlichkeitsberechnungen, Bewertung der Planungsergebnisse hinsichtlich späterer Nutzungskosten und deren Reduktion, Simulation von Nutzungsszenarien an virtuellen Facilities (Gebäude, Anlagen,...) Erarbeitung von Betreiberkonzepten sowie von Objektdokumentationssystemen usw. wesentlich zur Steigerung der Effektivität der Planungsergebnisse beitragen. Der Facility Manager zeichnet verantwortlich für die Entwicklung und Pflege der integrierten Datenbasis der Facilities, die eine wichtige Grundlage für die bedarfsgerechte Erfüllung der FMLeitungsvorgaben darstellt.

3. FM steht immer mehr für eine neue Dienstleistungskultur mit kompromißloser Kundenorientierung; ermöglicht die Konzentration auf das Kerngeschäft und fördert das Outsourcing von Hilfs- und Nebenprozessen (Bild 10).

Produktivitätssteigerung, Kostensenkung und gelebtes Qualitäts- und Umweltmanagement sind Leistungsprämissen der FM-Organisation. Dies bedeutet gleichzeitig die Bewältigung vielfältiger sozialer und technischer Herausforderungen durch die Führungskräfte im FM. Es darf nicht übersehen werden,

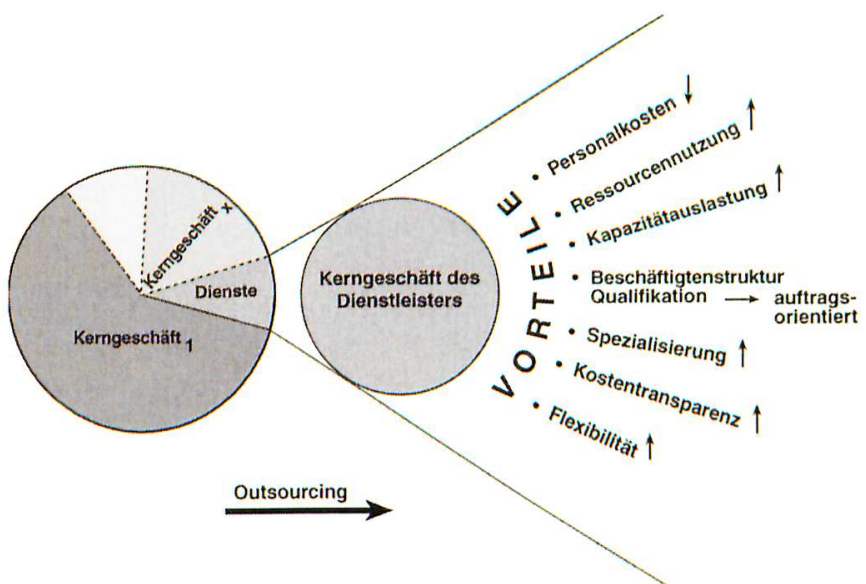

Bild 10: Vorteile des Outsourcing von FM-Dienstleistungen

daß mit der Einführung von FM in Unternehmen eine durchgreifende Rationalisierung der Organisation in der Bewirtschaftung und Verwaltung von Liegenschaften, Gebäuden und Anlagen erfolgt, die vielfach mit einem erheblichen Personalabbau verbunden ist. Gleichzeitig steigen die Qualifikationsanforderungen an die Beschäftigten bei der FM-Leistungserbringung. Die FM-Organisation muß den Technologiesprüngen der Informations-, Kommunikations- und Automatisierungstechnik sowie den sich verändernden Anspruchshaltungen der Kunden und damit den notwendigen Anpassungen der Facilities folgen können.

4. Der wachsende Automatisierungsgrad der Gebäude und Anlagen bis hin zur "künstlichen Intelligenz" verlangt nach Servicekonzepten, die dem Nutzer die

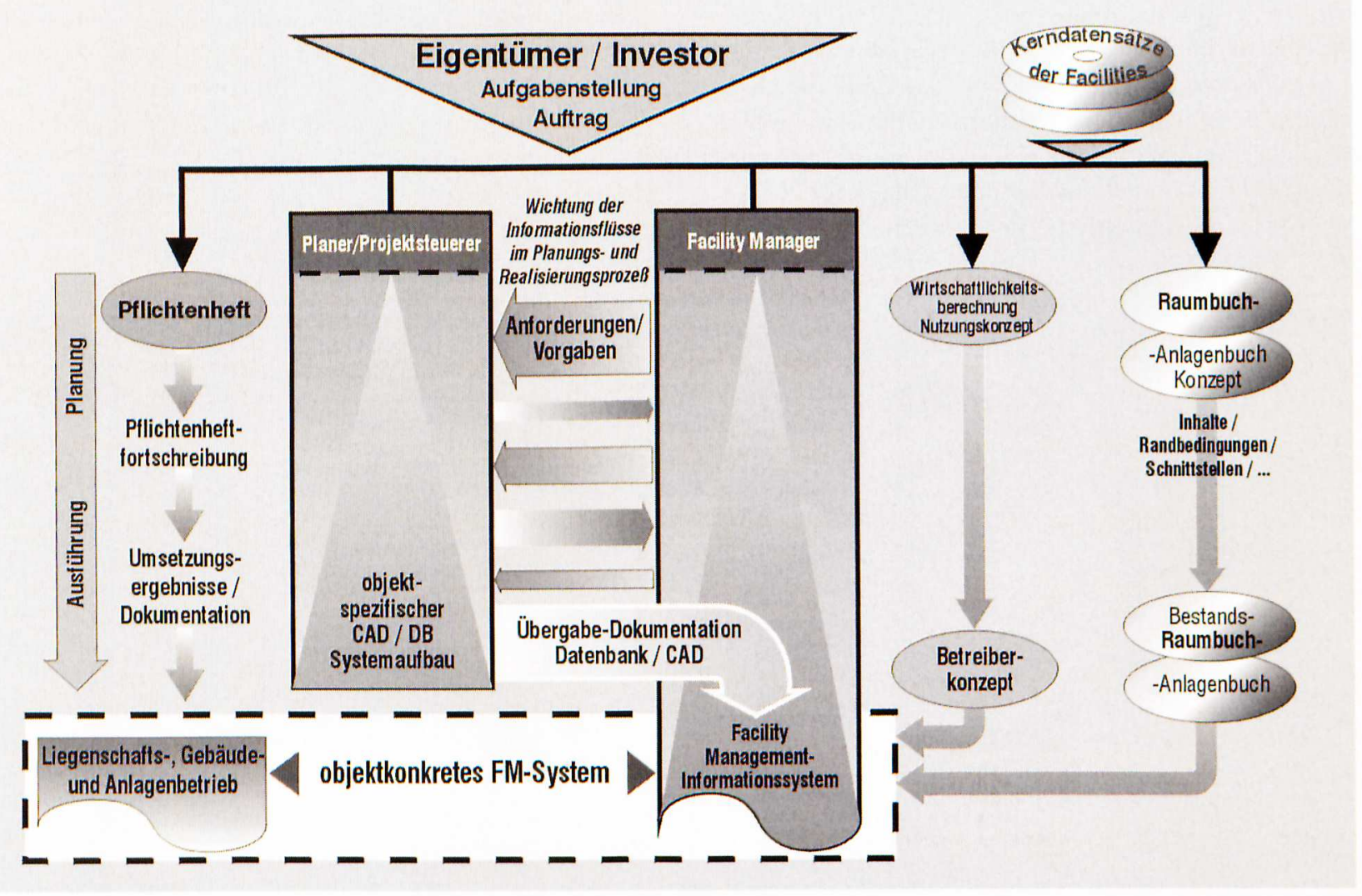

Bild 9: FM im Planungsprozeß 
den Facilities innewohnenden baulichen und technischen Möglichkeiten erschließen helfen. Kundenbedürfnisorientierter IT-Immobilienservice entwikkelt sich zum Standardangebot im Bereich des FM Online-Servicedienste erschließen den Nutzern einen neuen Komfort der wiederum bei diesen einen Produktivitätschub generiert.

5. Anbieter von integrierten FM-Leistungen werden in Zukunft den Markt dominieren. Integriertes FM steht für eine komplexe FM-Angebots- bzw. Leistungsform bei der ganzheitlich über den Lebenszyklus der Facilities hinweg FM in die jeweiligen Planungs- und Nutzungsprozesse integriert ist. Integriertes FM focusiert die Interessen der Eigentuimer, Nutzer und Betreiber auf die Maximierung des Nutzens aus den Facilities und gewährleistet die Optimierung der Aufbau- und Ablauforganisation in den FM-Leistungsprozessen. Große Unternehmungen, insbesondere solche in Konzernstruturen werden ihre verteilten FM-Leistungskompetenzen weiterentwickeln bzw. ergänzen und jeweils aufgabenbezogen beim Kunden in vernetzten Strukturen zusammenführen. Die Anforderungen an die Mobilität und Flexibilität der Führungskräfte steigen sprunghaft. Diese Organisationen werden den Wettbewerb dominieren. Im übergeordneten Interesse der Gesamtunternehmung werden besonders im FM-Servicebereich auch zeitweise negative Ergebnisbeiträge hingenommen. Der Preisdruck am Markt nimmt drastisch zu.
Auch wenn heute kleinere Unternehmen mit Leistungszukauf komplexe Leistungsangebote unterbreiten (Bild 11), so besitzen die sogenannten KMU längerfristig nur dann eine Marktchance, wenn es ihnen gelingt, sich zu kunden- bzw. auftragsorientierten virtuellen Netzwerken zusammenzuschließen, mit denen integrierendes FM abgesichert werden kann. Mit dem gezielten Einsatz der Kernkompetenzen der Netzwerkteilnehmer gelingt es ihnen, flexibel und effizient die Kundenbedürfnisse zu befriedigen. Die Kreativität der beteiligten Unternehmen, ihr natürliches Streben dem Kunden „Zusatznutzen“ anzubieten, ist auf Dauer gesehen ein deutlicher Wettbewerbsvorteil mit dem sich auch am Markt ein entsprechender Preis durchsetzen läßt.

Das Management solcher „FM-Leistungsnetze“ ist sicherlich sehr kompliziert und Erfahrungen müssen erst gesammelt werden. Dennoch gilt es bereits jetzt, die Auszubildenden an der Hochschule fuir diese spezifische Führungstätigkeit vorzubereiten.

6. Die beschriebenen Entwicklungstendenzen sind unmittelbar und aus vielfachen Ursachen heraus (Bild 12) mit steigenden Qualitätsanforderungen an die FM-Prozesse und die Dienstleister verbunden.

Gleichzeitig bewirkt FM in den Unternehmungen mit seinen Schnittstellen zu den am Kerngeschäft orientierten Qualitäts- und Umweltmanagementsystemen eine spürbare Unterstuitzung der damit verbundenen wirtschaftlichen Zielstellungen.

Facility Management- Komplexangebot (Beispiel)

\begin{tabular}{|c|c|c|c|}
\hline Beratung & Beschaffu & Durchführung & Controlling \\
\hline Flächen-Bewirtschaftung & Kaufmännische Bewirtschaftung & Technische Bewirtschaftung & Sonstige Dienstleistungen \\
\hline $\begin{array}{l}\text { Flächen- / Raumplanung } \\
\text { - Immobilien- / Standortbewertung } \\
\text { - Flächenorganisation } \\
\text { - Arbeitsplatzanalyse } \\
\text { Bestandserfassung / -pflege } \\
\text { - Flächeninventarisierung / -pflege } \\
\text { - Inventarerfassung / -pflege } \\
\text { - Anlageninventarisierung / -pflege } \\
\text { - Raumbucherstellung / -pflege } \\
\text { - Dispositions- Flächenreserven } \\
\text { - Zentrale Dienste } \\
\text { - Gebäudesicherheit } \\
\text { - Telefang } \\
\text { - Reinigung } \\
\text { - Winterdienst } \\
\text { - Pflege der Außenanlage } \\
\text { - Gertenpflege } \\
\text { - Pflanzenpflege im Gebäude } \\
\text { - Schädlingsbekämpfung } \\
\text { - Poststelle /-service } \\
\text { - Reisestelle / -service } \\
\text { - Zentralsekretariat } \\
\text { - Fuhrparkverwaltung } \\
\text { - Kantinenbewirtschaftung } \\
\text { - Umzugsdienst } \\
\text { - Zentralarchiv } \\
\text { - Botendienste } \\
\text { - Druckerei } \\
\text { - Kopierdienst } \\
\text { - Büromaterial } \\
\text { - Sicherheitsfachkraft } \\
\text { - Medizinischer Dienst } \\
\text { - Wäscherei } \\
\text { - Büroservice } \\
\text { - EDV-Unterstütżung } \\
\text { - Entsorgung } \\
\text { - Tiefgaragenbetrieb }\end{array}$ & $\begin{array}{l}\text { Objekt-Steuerung } \\
\text { - Objekt-, Projekt-Management } \\
\text { - Nutzerbetreuung } \\
\text { - Eigentümerbetreuung } \\
\text { - Objektbetreuung } \\
\text { - Objektinspektion } \\
\text { - Budgetierung } \\
\text { - Kostenoptimierung } \\
\text { - Vermittlung von Dienstleistungen } \\
\text { Leistungskoordinierung } \\
\text { - Vertragspflichtenüberwachung } \\
\text { - Flëchenübergabe und -abnahme } \\
\text { - Betreuung von Nutzer- und } \\
\text { Werbegemeinschaften } \\
\text { - Center-Management } \\
\text { - Dokumentation / Berichtswesen } \\
\text { Rechnungswesen } \\
\text { - Kostenberechnung und - } \\
\text { abrechnung } \\
\text { - Inkesso u. a. Miet- und Neben- } \\
\text { kosten } \\
\text { - Kostenträger- und Kostenstellen- } \\
\text { zuordnung } \\
\text { - Miet- und flächenbezogene Buch- } \\
\text { haltung } \\
\text { - Lohn- und Finanzbuchhaltung } \\
\text { - Liquiditätsanalyse } \\
\text { - Mahnwesen } \\
\text { - Steuern u. a. Umsatzsteuervoranm. } \\
\text { Vertrags-, Versicherungs-, } \\
\text { Rechts- und Personalwesen } \\
\text { - Vertragsabschluß und Pflege } \\
\text { - Versicherungsabschluß und -pflege } \\
\text { - Vertrags- und Kautionsverwaltung } \\
\text { - Index-, Miet- und Nebenkosten- } \\
\text { anpassung } \\
\text { Vermietungswesen } \\
\text { - Vemietungskonzeption. Mietermix } \\
\text { - Erst-, Nach- und Untervermietung }\end{array}$ & $\begin{array}{l}\text { Instandhaltung } \\
\text { Wartung, Inspektion, Instand- } \\
\text { setzung, } \\
\text { Betriebsmittel, Stördienst } \\
\text { - Gastechnik } \\
\text { - Wassertechnik } \\
\text { - Abwassertechnik } \\
\text { - Wärmeversorgungstechnik } \\
\text { - Brauchwassererwärmungstechnik } \\
\text { - Raumluttechnik } \\
\text { - Kältetechnik } \\
\text { - Elektrotechnik } \\
\text { - Blitzschutz } \\
\text { - Autzugs-, Förder- und Lagertechnik } \\
\text { - kraftbetätigte Tür- und Toranlagen } \\
\text { - Sicherheitstechnik } \\
\text { - Mess-/ Steuer- / Regeltechnik } \\
\text { - Gebäudeautomation } \\
\text { - Nachrichten und Kommunikations- } \\
\text { technik } \\
\text { - Kabel- und Netzwerktechnik } \\
\text { - EDV-Technik } \\
\text { EDV-Sicherheitstechnik } \\
\text { - Wäscherei- und chemische } \\
\text { - Reinigungstechnik } \\
\text { - Müchentechnik } \\
\text { - Medizin- und Labortechnik } \\
\text { - Bürogereitechnik } \\
\text { - sonstige Technik } \\
\text { Technische Betriebsführung } \\
\text { - Haustechniker / Hauswart } \\
\text { - 24-h Service und } \\
\text { - Bereitschaftsdienst } \\
\text { und Zentralen } \\
\text { - Zentrale Leittechnik } \\
\text { - Energie-Management } \\
\text { - Kabel- und Netzwerkmanagement }\end{array}$ & $\begin{array}{l}\text { - Erstellung von Bewirtschaftungs- } \\
\text { konzepten bei Projektplanungen und } \\
\text { bestehenden Organisationen, } \\
\text { Leistungsverzeichnisse, } \\
\text { Ausschreibung, Vergabe, } \\
\text { Dokumentation, Überwachung der } \\
\text { Durchführung } \\
\text { - Bewirtschaftungskostenberechnung } \\
\text { bei bestehenden Gebäuden, } \\
\text { geplanten Neubauten, Umbauten, } \\
\text { Renovierungen, Sanierungen, } \\
\text { Revitalisierungen } \\
\text { - Wirtschaftlichkeitsbetrachtungen für } \\
\text { Immobiliennutzung } \\
\text { - FM-Auswertung für Architekten und } \\
\text { Fachingenieurleistungen } \\
\text { - Energieberatung } \\
\text { - Performance Contracting } \\
\text { - Gebäude- und Nutzungssimulation } \\
\text { - CAFM-Consulting }\end{array}$ \\
\hline
\end{tabular}




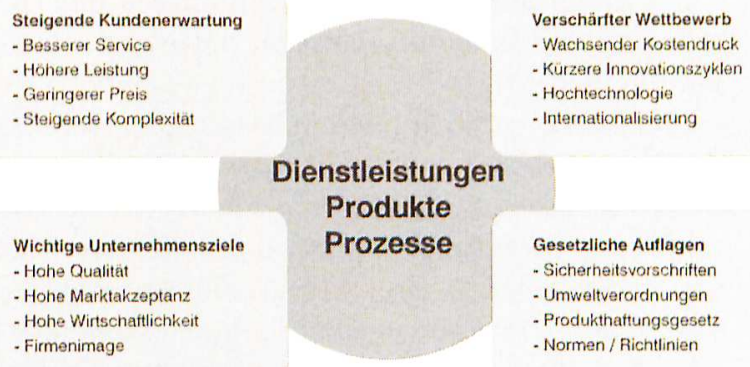

Bild 12: Ursachen für steigende Qualitätsanforderungen im Dienstleistungsbereich [4]

\section{Aufbau eines Lehrgebietes für integriertes FM}

Fïr wen produziert die Hochschule? Diese Frage aus der Sicht des FM-Studiengangs zu beantworten ist nicht leicht, da wie ausgefuihrt ein großer Markt mit breitem Einsatzspektrum der Absolventen zu bedienen ist. Stark vereinfacht gesagt - für selbständige bzw. in Konzernstrukturen eingebundene FM-Organisationen (Unternehmen).

Welche Fähigkeiten des „Produkts“ erwarten diese Unternehmen? Sie erwarten in erster Linie den Generalisten mit sogenannten "Schlïsselqualifikationen" wie Sozialkompetenz und Kundenorientierung, Führungswille, Teamfähigkeit, souveräner Umgang mit Daten, Datenverarbeitungs- und Kommunikationstechniken, Fähigkeit zu strategischem und problem- bzw. prozeßorientiertem Denken und Handeln. Diese Fähigkeiten sind nicht in Einzelseminaren zu vermitteln, sondern nur im Rahmen des gesamten Studienganges.

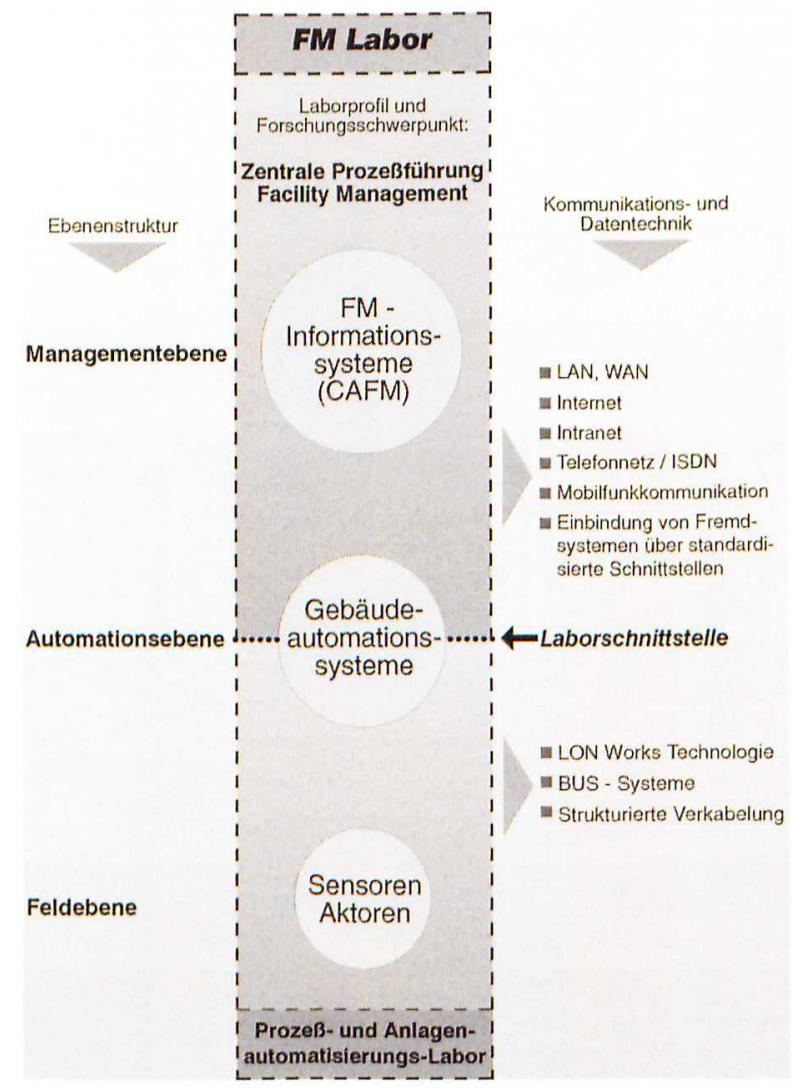

Bild 14: FM-Laborverbundkonzept

\begin{tabular}{|c|c|}
\hline $\begin{array}{l}\text { Studiengang: } \\
\text { Studienabschnitt: } \\
\text { Studienrichtung: }\end{array}$ & $\begin{array}{l}\text { Wirtschaftsingenieurwesen } \\
\text { Hauptstudium } \\
\text { Facility Management }\end{array}$ \\
\hline \multicolumn{2}{|l|}{ STUDIENFÄCHER } \\
\hline \multicolumn{2}{|l|}{ Einführung in FM } \\
\hline \multicolumn{2}{|c|}{$\begin{array}{l}\text { Technische Grundlagen } \\
\text { Technische Gebäudeausrüstung } \\
\text { Informations- und Kommunikationssysteme }\end{array}$} \\
\hline \multicolumn{2}{|c|}{$\begin{array}{l}\text { Immobilienmanagement } \\
\text { Immobilienmanagement I } \\
\text { Immobilienmanagement II }\end{array}$} \\
\hline \multicolumn{2}{|c|}{$\begin{array}{l}\text { Technisches Management } \\
\text { Gebäudeautomatisierung / } \\
\text { Computerintegriertes Gebäude } \\
\text { Anlagenbetrieb und Instandhaltungsorganisation } \\
\text { Energiemanagement }\end{array}$} \\
\hline \multicolumn{2}{|c|}{$\begin{array}{l}\text { Kaufmännisches Management } \\
\text { FM- Kostenrechnung und Controlling } \\
\text { FM- Projekt- und Vertragsmanagement } \\
\text { Facilities- Marketing }\end{array}$} \\
\hline \multicolumn{2}{|c|}{$\begin{array}{l}\text { Infrastrukturelles Management } \\
\text { Flächen- und Umzugsmanagement } \\
\text { Infrastrukturdienste }\end{array}$} \\
\hline \multicolumn{2}{|c|}{$\begin{array}{l}\text { Rechnergestütztes FM } \\
\text { Computer Aided Facility Management (CAFM) } \\
\text { Rechnergestützte Bestandsdatenerfassung / } \\
\text { Dokumentationsmanagement }\end{array}$} \\
\hline \multicolumn{2}{|c|}{$\begin{array}{l}\text { Integrierte Managementsysteme im FM } \\
\text { Qualität/Umwelt/Sicherheit }\end{array}$} \\
\hline \multicolumn{2}{|c|}{ Volkswirtschaftslehre } \\
\hline \multicolumn{2}{|c|}{$\begin{array}{l}\text { Ergänzende Qualifikation } \\
\text { Steuerrecht } \\
\text { Personalführung } \\
\text { Verhandlungsf./Rhetorik } \\
\text { Fremdsprachen II }\end{array}$} \\
\hline \multicolumn{2}{|c|}{ Projektstudium FM } \\
\hline $\begin{array}{l}\text { Wahlpflichtfächeı } \\
\text { Networkman } \\
\text { Flughafenma } \\
\text { Krankenhaus }\end{array}$ & $\begin{array}{l}\text { agement } \\
\text { nagement } \\
\text { management }\end{array}$ \\
\hline
\end{tabular}

Bild 13: Studienfächer im FM-Hauptstudium

Die fuir die FM-Ausbildung geschaffene Studienordnung der TFH Wildau (Bild 13) verfolgt dieses Ziel, wobei die Dynamik der Entwicklung des Marktes natürlich deren ständige Weiterentwicklung erfordert.

Facility Management gehört mit zu den Disziplinen, bei denen es immer schwieriger wird, die Studierenden mit einem deutlichen Vorhalt der Wissensvermittlung auf den Eintritt in die FM-Organisationen vorzubereiten. Um so wichtiger ist es, daß in allen Einzeldisziplinen konsequent die fortgeschrittensten theoretischen und praktischen Erkenntnisse und Fertigkeiten vermittelt werden. Die FM-Ausbildung an der TFH Wildau besitzt eine stark praxisbezogene Orientierung. Dazu wurde gemeinsam mit dem Fachgebiet Prozeß-/Anlagenautomatisierung eine Laborstruktur geschaffen (Bild 14), die diese deutlich widerspiegelt.

Das im Aufbau befindliche FM-Labor wird an den Aufgaben des „FM-Fernmanagement“ ausgerichtet d.h. damit kann im Labor einem der wichtigsten FM-Trends gefolgt und konkrete Projektarbeit geleistet werden. 

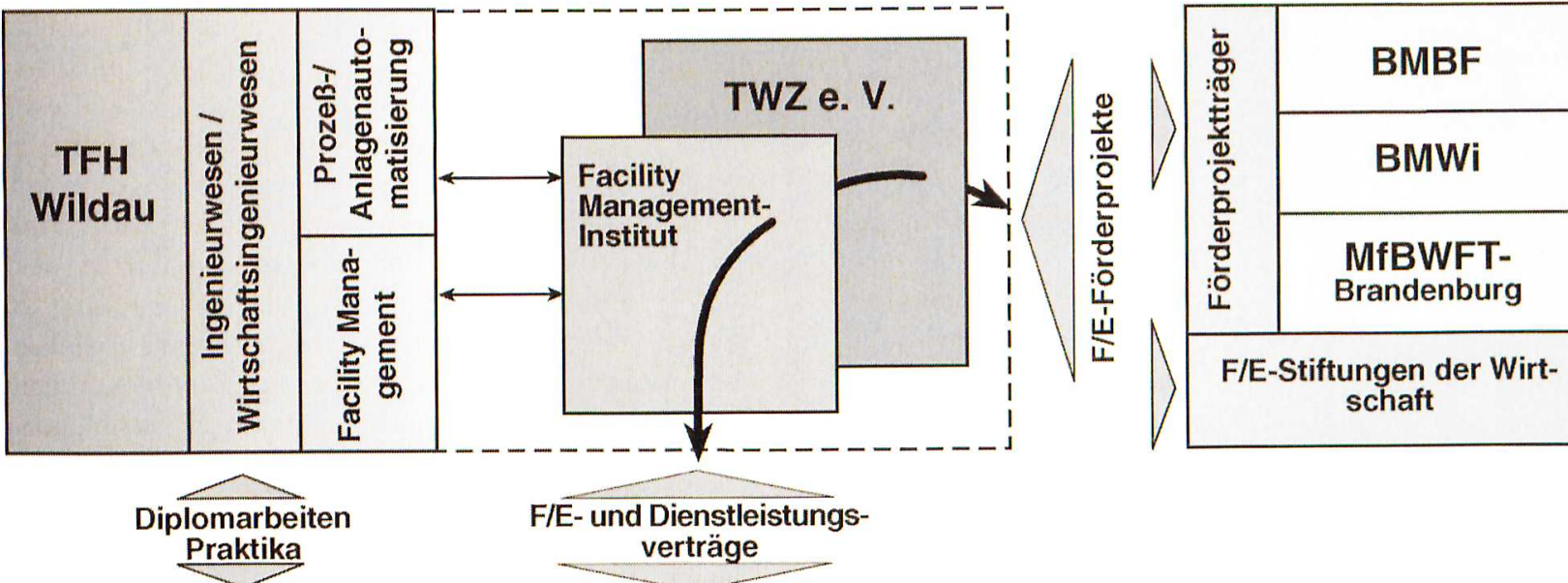

F/E- und Dienstleistungs-

verträge

angestrebte Leistungs- und Partnerbeziehungen
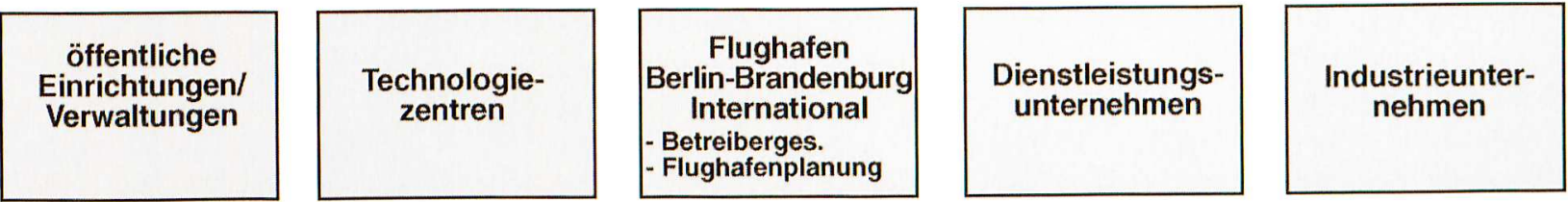

Bild 15: Beziehungsorganigramm des FM-Instituts am TWZ

Projektarbeit, Praktika, Diplomarbeiten usw. an Entwicklungsschwerpunkten des FM auszurichten, ist ein wesentlicher Grundsatz der Ausbildung, zu dem auch das kuirzlich am Technologietransfer- und Weiterbildungszentrum an der Technischen Fachhochschule Wildau e.V. geschaffene „Institut für Facility Management“ einen entscheidenden Beitrag leisten wird (Bild 15).

Das Institut, als ein zukïnftiges FM-Kompetenzzentrum in der Region, wird sich vorrangig

- der Ausarbeitung von Strategien und Methoden für Problemlösungsprozesse des FM in öffentlichen Bereichen und der Wirtschaft (Industrial FM);

- den zukünftigen Aufgaben des technischen FM in sog. ,intelligenten“ Gebäuden und Anlagen;

- rechnergestuitzten Methoden des FM (CAFM) im Zusammenhang mit multimedialer Kommunikation und virtueller Objektabbildung;

widmen.

Der Erfolg wird wesentlich dadurch bestimmt sein, wie es gelingt, enge Partnerbeziehungen zur öffentlichen Verwaltung und zur Wirtschaft zu kniipfen.

\section{Zusammenfassung}

Mit dem Hauptstudiengang Facility Management am Fachbereich Ingenieurwesen/ Wirtschaftsingenieurwesen erfüllt die Technische Fachhochschule Wildau als eine der ersten in der Bundesrepublik Deutschland das vom Deutschen Verband für Facility Management GEFMA e.V. ausgearbeitete Anforderungsprofil für die Hochschulausbildung. Mit dem klaren Bekenntnis zur Ausbildung von Führungskräften für FM und der Orientierung an den maßgeblichen Entwicklungstrends wird gleichfalls den Erwartungen der Wirtschaft an die Absolventen entsprochen. Starker Praxisbezug, intensive
Laborarbeit und die Einbeziehung der Studenten in die Aufgaben des FM-Instituts sichern den Auszubildenden gute Chancen für ihren Eintritt in Unternehmungen des FM-Angebots- und Nachfragemarktes.

1 Zeitabschnitt zwischen Idee, Planung, Realisierung, Nutzung, ggf. Umbau und Umnutzung bis zum Rückbau und Recycling von Facilities

2 Sammelbegriff für Liegenschaften, Gebäude, bauliche und technische Anlagen; auch als Synonym für Anlagen allgemein bzw. im Zusammenhang mit dem Begriff Anlagenwirtschaft

3 Major Players - Gruppe der Hauptbeteiligten

\section{Literatur}

[1] GEFMA e.V., Richtlinie Nr. 610 „FM-Studiengänge“, 1998

[2] Bleicher, K., Das Konzept Integriertes Management. Frankfurt/New York 1992

[3] Frutig, D., Reiblich,D., Facility Management: Objekte erfolgreich verwalten und bewirtschaften. Zürich 1995

[4] Brakhahn, W., Vogt, U., ISO 9000 für Dienstleister. Landsberg 1996

\section{Autor}

Prof. Dr.-Ing. Dietrich Reiblich

Technische Fachhochschule Wildau

Fachbereich Ingenieur-/Wirtschaftsingenieurwesen

Bahnhofstraße

15745 Wildau

Tel. (0 33 75) 508-115

oder (0 30) 6576-2204

E-Mail: dreib@igw.tfh-wildau.de

oder: reiblich@ipw-berlin.de 